\title{
Difference Schemes for Degenerate Parabolic Equations
}

\author{
By E. A. Socolovsky*
}

\begin{abstract}
Diagonal dominant implicit-difference schemes approximating a porous media type class of multidimensional nonlinear equations are shown to generate semigroups in an approximate $L^{1}$-space, and the rate of convergence to the semigroup solution in $L^{1}$ is given. The numerical schemes proposed by Berger et al. in [4] are described and a proof of convergence for the fully discrete algorithms is outlined. Numerical experiments are discussed.
\end{abstract}

1. Introduction. In this paper we consider difference schemes for the problem

$$
\begin{array}{lrl}
u_{t}-\Delta f(u)+g(u)-a u=0, & t>0, & x \in \Omega, \\
u(t, x)=0, & x \in \partial \Omega, \\
u(0, x)=u_{0}(x), & x \in \Omega .
\end{array}
$$

Here, $f$ is continuous monotone increasing, $g$ is monotone nondecreasing, $a \geqslant 0$, and $f(0)=g(0)=0$. We also assume that $f^{-1}$ and $g$ are Hölder continuous with exponents $\alpha$ and $\beta$, respectively. (P) is a parabolic problem that degenerates when $u=0$. It is found, for instance, in the diffusion through a porous medium and in some population models [17]. In the porous medium case, $g(u)=a=0$ with $f(u)=u^{m}, m>1$, and for Lotka's population model, $g(u)=K u^{2}, a>0$, with $f(u)=u^{m}, m \geqslant 2$.

Our difference schemes are patterned on the nonlinear semigroup theory for (P) [1], [10]. We carry the analysis for one particular scheme and sketch its extension to schemes obtained from other diagonal dominant discretizations of the Laplacian. We concentrate on the case $a=0$, which involves the essential techniques, and outline the case $a>0$. The details of the case $a>0$ and the nonhomogeneous equation are considered in [25, Chapter I]. We discretize in space and prove that the resulting operator generates a nonlinear semigroup $S^{h}(t)$ in a discrete $L^{1}$-space. $S^{h}(t)$ is a discrete analog of $S(t)$, the semigroup of operators in $L^{1}(\Omega)$ generated by the space operator in (P) and, in a sense to be made precise, we show that for any $u_{0} \in L^{1}(\Omega)$, " $S^{h}(t) u_{0} \rightarrow S(t) u_{0}$ as $h \rightarrow 0$ ".

There are a number of papers discussing difference schemes for problems like (P) when $g \equiv 0$ and $a=0$, e.g., [4], [11], [13], [18], [21]. In Section 5 we consider the schemes proposed in [4], which also arise from a semigroup approach to (P). The

Received December 2, 1983; revised April 22, 1985.

1980 Mathematics Subject Classification. Primary 65M10, 65J15.

Key words and phrases. Difference schemes, degenerate nonlinear parabolic equations, nonlinear semigroups.

*Current address: Department of Mathematics, University of Connecticut, Storrs, CT 06268. 
analytical algorithm consists of discretizing in time and approximating the generator equation. Its convergence was established in [4]. Here we outline a proof based on our results, for the fully discrete schemes.

An important manifestation of the degeneracy of $(\mathrm{P})$ is that if $u_{0}$ is positive of compact support, so is the solution $u$ of (P) for all $t>0$ [12]. Our method also has finite speed of propagation, and in Section 6 we discuss numerical experiments conducted with both methods for a known exact solution of compact support [2], [23]. In this case, our chosen scheme was more accurate, especially in determining the interface, but not as accurate as the one-dimensional schemes in [18], [21] which are specifically designed to track the interface.

The scheme in [11] is essentially the same as our sample scheme. It is proven there that the limit of the difference solutions yields a weak solution of (P). As indicated in [11], those proofs hold for $\Omega$ one-dimensional or a rectangle. In contrast, our proofs are valid for more general regions and grids. We also show that the rate of convergence to the semigroup solution of $(\mathrm{P})$ for smooth initial data is $O\left(\Delta t^{1 / 2}\right)+$ $O\left(h^{\alpha \beta /(3+2 \alpha)}\right)$. The computed rates were $O(\Delta t)$.

Finite element discretizations can be found in [24] for a problem similar to (P), and in [19], [25] for a Lagrangian formulation of the porous media problem.

2. Preliminary Results. Let $\Omega \subset \mathbf{R}^{d}$ be a bounded domain with $C^{2, \alpha \beta}$ boundary, and $\|v\|_{p}$ denote the $L^{p}(\Omega)$-norm of $v$. An operator $A$ with domain $D(A)$ belongs to the class $\mathscr{A}(a)$ for $a>0$ if for each $0<\lambda<a^{-1}$ and any $x, y \in D(A)$ :

$$
\|(x+\lambda A x)-(y+\lambda A y)\| \geqslant(1-\lambda a)\|x-y\| \text {. }
$$

Let us now define the operator $A$ by $A u:=-\Delta f(u)+g(u)-a u$ with domain $D(A)=\left\{u \in L^{1}(\Omega): f(u) \in W_{0}^{1,1}(\Omega), g(u) \in L^{1}(\Omega)\right.$ and $-\Delta f(u) \in L^{1}(\Omega)$ in weak sense $\}$. Let $\gamma_{\lambda}$ be defined by $\gamma_{\lambda}(x):=f^{-1}(x)+\lambda g \circ f^{-1}(x)$; then the assumptions made on $f$ and $g$ imply that for any $\lambda>0, \gamma_{\lambda}$ has a maximal monotone graph. It follows that $A$ has the following properties:

LEMmA 2.1. (i) $A \in \mathscr{A}(a)$ in $L^{1}(\Omega)$, and $\operatorname{Range}(I+\lambda A)=L^{1}(\Omega)$, i.e., for $0<\lambda<a^{-1}$ the resolvent operator $J_{\lambda}:=(I+\lambda A)^{-1}$ is Lipschitz continuous with constant $(1-\lambda a)^{-1}$ and $D\left(J_{\lambda}\right)=L^{1}(\Omega)$.

(ii) $D(A)$ is dense in $L^{1}(\Omega)$.

(iii) A generates a nonlinear semigroup $S(t)$ on $L^{1}(\Omega)$ and $u(t):=S(t) u_{0}$ is a solution of $(\mathrm{P})$ in the semigroup sense.

(iv) Let $v \in L^{p}(\Omega), 1 \leqslant p \leqslant \infty$; then $\left\|J_{\lambda} v\right\|_{p} \leqslant(1-\lambda a)^{-1}\|v\|_{p}$.

(v) Let $v, \bar{v} \in L^{1}(\Omega)$; also let $u:=J_{\lambda}(v)$ and $\bar{u}:=J_{\lambda} \bar{v}$; then $\left\|[u-\bar{u}]^{+}\right\|_{1} \leqslant$ $\left\|[v-\bar{v}]^{+}\right\|_{1}$. Consequently, if $v \leqslant \bar{v}$ a.e., then $u \leqslant \bar{u}$ and $f(u) \leqslant f(\bar{u})$ a.e.

Proof. First consider the case where $g \equiv 0$ and $a=0$. Brezis and Strauss established statements (i), (iv), and (v) in [7, Theorem 1, Propositions 4 and 5]. Benilan also established (i) in [3, Chap. II, Théorème 2.1]. Assertion (ii) is a known result and can be found for instance in [15, Proposition 1]. Statement (iii) follows from the Generation Theorem of Crandall and Liggett [8] and the general theory of nonlinear semigroups, see [1]. Statement (i) can also be found in [10, Theorem 4.12].

Most of these statements were established by considering the problem $\gamma(w)-$ $\lambda \Delta w=v$ for $w \in D(-\Delta):=\left\{u \in W_{0}^{1,1}(\Omega):-\Delta u \in L^{1}(\Omega)\right.$ in weak sense $\}$ and defining $J_{\lambda} v:=\gamma(w)$. In the case $g \not \equiv 0$ and $a=0, J_{\lambda}$ is defined as $J_{\lambda} v:=f^{-1}(w)$, 
and the statements easily follow from the monotonicity of $g$ and $g(0)=0$. Finally, the case $a>0$ is established by a simple fixed-point argument (see [25]).

3. Approximate $L^{1}$-Spaces and Difference Schemes. We construct either a full or a uniform degree-zero grid approximation and denote the gridpoints by $x_{i}, i$ being their corresponding index. We recall that in a full grid the nodes are given by the intersection of lines parallel to the axis with themselves or the boundary. In a degree-zero grid the boundary nodes are the interior intersection points closest to the boundary. Grid functions are indicated with a subscript $h$, and $v_{i}$ denotes the value of $v_{h}$ at $x_{i}$.

In one dimension we let $h_{i}:=x_{i}-x_{i-1}$ and $h:=\max _{i} h_{i} \cdot Q_{i}$ is the interval around $x_{i}$ given by

$$
Q_{i}:=\left\{x:-\frac{1}{2} h_{i} \leqslant x-x_{i}<\frac{1}{2} h_{i+1}\right\}
$$

with volume $w_{i}=\frac{1}{2}\left(h_{i}+h_{i+1}\right)$. In the $\mathbf{R}^{d}$ case, $i$ and $h_{i}$ are vectors, $Q_{i}=Q_{i_{1}} \times Q_{i_{2}}$ $\times \cdots \times Q_{i_{d}}$ with $w_{i}=w_{i_{1}} w_{i_{2}} \cdots w_{i_{d}}$.

We denote by $l_{h}^{1}$ the space of grid functions defined over the interior points of the grid normed by $\left\|v_{h}\right\|_{h}:=\sum w_{i}\left|v_{i}\right|$. A restriction operator $r_{h}: C(\Omega) \rightarrow l_{h}^{1}$ is defined by $\left(r_{h} v\right)_{i}:=v\left(x_{i}\right)$, and a prolongation operator $p_{h}: l_{h}^{1} \rightarrow L^{1}(\Omega)$ by $p_{h} v_{h}:=\sum_{i} v_{i} \chi_{Q_{i}}$, where $\chi_{Q}$, denotes the characteristic function of $Q_{i}$. It is clear that for any Riemann integrable $v,\left\|v-p_{h} r_{h} v\right\|_{1} \rightarrow 0$ as $h \rightarrow 0$; also as $\left\{Q_{i}\right\}$ are disjoint,

$$
\int_{\Omega}\left|\sum_{i} v_{i} \chi_{Q_{i}}\right|=\sum_{i}\left|v_{i}\right|\left|Q_{i} \cap \Omega\right| \leqslant \sum_{i} w_{i}\left|v_{i}\right| \quad \text { or }\left\|p_{h} v_{h}\right\|_{1} \leqslant\left\|v_{h}\right\|_{h} .
$$

Our finite-difference analog of $(\mathrm{P})$ is

$$
\begin{array}{r}
\left(u_{i}^{n}-u_{i}^{n-1}\right) / \Delta t-\Delta_{h} f\left(u_{i}^{n}\right)+g\left(u_{i}^{n}\right)-a u_{i}^{n}=0 \\
\text { for } x_{i} \text { an interior point of the grid, } \\
u_{h}^{0}=r_{h} v_{0}, \quad v_{0} \in C^{0, \alpha \beta}(\bar{\Omega}) \text { approximates } u_{0} .
\end{array}
$$

Here the superscript $n$ denotes the discrete time level and $\Delta_{h}$ is a diagonally dominant scheme approximating the Laplacian. We describe this family of schemes below.

Let $L_{h}$ be a scheme consistent with the linear differential operator $L$, and let $I$ denote the family of indices of gridpoints at which we apply $L_{h}$. Also let $v_{h}$ be a grid function satisfying $v_{i}=0$ for $i \notin I$. We have

$$
\sum_{i \in I} w_{i} L_{h} v_{i}=\sum_{i \in I} a_{i} v_{i}, \quad a_{i}=\sum_{k \in I} w_{k} b_{i k}
$$

where $b_{i k}$ is the coefficient of $u_{i}$ in the term $L_{h} v_{k}$. We say that $L_{h}$ is diagonally dominant if

$$
w_{i} b_{i i} \geqslant \sum_{k \neq i} w_{k}\left|b_{i k}\right|
$$

For simplicity we shall consider the case when $\Delta_{h}$ is the usual difference approximation of the Laplacian, which in one space dimension is given by

$$
-\Delta_{h} v_{i}:=\frac{2}{\left(h_{i}+h_{i+1}\right)}\left\{\left(\frac{1}{h_{i}}+\frac{1}{h_{i+1}}\right) v_{i}-\frac{1}{h_{i}} v_{i-1}-\frac{1}{h_{i+1}} v_{i+1}\right\},
$$


where, whenever $i-1$ or $i+1$ indicate boundary nodes, we substitute $v_{i-1}$ or $v_{i+1}$ by the boundary value at the node, which in this case is zero. In $\mathbf{R}^{d}, \Delta_{h}:=\sum_{j=1}^{d} \Delta_{h, j}$, where $\Delta_{h, j}$ is the above discrete Laplacian taken in the $j$ th direction.

Let $k=\Delta t$. We can rewrite $\left(\mathrm{P}_{h}\right)$ as

$$
\left(I+k A_{h}\right) u_{h}^{n}=u_{h}^{n-1}, \quad u_{h}^{0}=r_{h} v_{0},
$$

with the operator $A_{h} \subset l_{h}^{1} \times l_{h}^{1}$ defined by

$$
\left(A_{h} v_{h}\right)_{i}:=-\Delta_{h} f\left(v_{i}\right)+g\left(v_{i}\right)-a v_{i} .
$$

TheOREM 3.1. $A_{h} \in \mathscr{A}(a)$ and Range $\left(I+\lambda A_{h}\right)=l_{h}^{1}$, i.e., for $0<\lambda<a^{-1}$ the resolvent operator $J_{\lambda}^{h}:=\left(I+\lambda A_{h}\right)^{-1}$ is Lipschitz continuous with constant $(1-\lambda a)^{-1}$, and its domain is all of $l_{h}^{1}$.

Proof. First consider the case $a=0$, i.e., we show that $A_{h}$ is $m$-accretive. Let $u_{h}, v_{h} \in l_{h}^{1}$, and define the sets of indices $I^{+}$and $I^{-}$by

$$
\begin{aligned}
& I^{+}:=\left\{i: u_{i} \geqslant v_{i} \text { and } x_{i} \text { an interior point of the grid }\right\}, \\
& I^{-}:=\left\{i: u_{i}<v_{i} \text { and } x_{i} \text { an interior point of the grid }\right\} .
\end{aligned}
$$

Let $\lambda>0$; then as $g$ is monotone nondecreasing,

$$
\begin{aligned}
\|\left(I+\lambda A_{h}\right) u_{h} & -\left(I+\lambda A_{h}\right) v_{h} \|_{h} \\
= & \sum_{i \in I^{+} \cup I^{-}} w_{i}\left|u_{i}-v_{i}-\lambda \Delta_{h}\left[f\left(u_{i}\right)-f\left(v_{i}\right)\right]+g\left(u_{i}\right)-g\left(v_{i}\right)\right| \\
\geqslant & \sum_{i \in I^{+}} w_{i}\left(u_{i}-v_{i}-\lambda \Delta_{h}\left[f\left(u_{i}\right)-f\left(v_{i}\right)\right]\right) \\
& +\sum_{i \in I^{-}} w_{i}\left(v_{i}-u_{i}-\lambda \Delta_{h}\left[f\left(v_{i}\right)-f\left(u_{i}\right)\right]\right) .
\end{aligned}
$$

As $f$ is monotone increasing, $\left[f\left(u_{i}\right)-f\left(v_{i}\right)\right] \geqslant 0$ when $i \in I^{+}$, and $\left[f\left(v_{i}\right)-f\left(u_{i}\right)\right]$ $\geqslant 0$ when $i \in I^{-}$. Hence, in one space dimension with a uniform grid we obtain

$$
\begin{aligned}
\sum_{i \in I^{+}} w_{i}\left(-\Delta_{h}\right)\left[f\left(u_{i}\right)-f\left(v_{i}\right)\right]+\sum_{i \in I^{-}} w_{i}\left(-\Delta_{h}\right)\left[f\left(v_{i}\right)-f\left(u_{i}\right)\right] \\
=\sum_{i \in I^{+}} \frac{1}{h}\left\{2\left[f\left(u_{i}\right)-f\left(v_{i}\right)\right]-\left[f\left(u_{i-1}\right)-f\left(v_{i-1}\right)\right]-\left[f\left(u_{i+1}\right)-f\left(v_{i+1}\right)\right]\right\} \\
\quad+\sum_{i \in I^{-}} \frac{1}{h}\left\{2\left[f\left(v_{i}\right)-f\left(u_{i}\right)\right]-\left[f\left(v_{i-1}\right)-f\left(u_{i-1}\right)\right]-\left[f\left(v_{i+1}\right)-f\left(u_{i+1}\right)\right]\right\} \\
=\sum_{i \in I^{+} \cup I^{-}} \frac{1}{h}[2 \pm 1 \pm 1]\left|f\left(u_{i}\right)-f\left(v_{i}\right)\right| \geqslant 0 .
\end{aligned}
$$

For a nonuniform grid the coefficient above is replaced by

$$
\left[\frac{1}{h_{i}}+\frac{1}{h_{i+1}} \pm \frac{1}{h_{i}} \pm \frac{1}{h_{i+1}}\right] \geqslant 0 .
$$

In the $\mathbf{R}^{d}$ case, for each $\Delta_{h, j}$ we sum over the $j$ th index first to obtain the same expression multiplied by $\left(\Pi_{l \neq j} w_{i}\right)$ and summed over all other indices. In general, for any diagonal dominant scheme the coefficient of $\left|f\left(u_{i}\right)-f\left(v_{i}\right)\right|$ is not less than $w_{i} b_{i i}-\sum_{k \neq i} w_{k}\left|b_{i k}\right| \geqslant 0$. Finally, substituting the above in (3.2) yields accretiveness; 
$m$-accretiveness follows from the fact that for fixed $h, A_{h}$ is continuous [1], [20]. The case $a>0$ is established by a simple fixed-point argument (see [25]).

Remark 3.2. From (3.1) it follows that the solution of $\left(\mathrm{P}_{h}\right)$ is given by

$$
u_{h}^{n}=\left(J_{k}^{h}\right)^{n} r_{h} v_{0},
$$

and Theorem 3.1 shows that (3.3) has a unique solution and is stable.

The next lemma is a maximum principle type result for $\left(\mathrm{P}_{h}\right)$ when $\Delta_{h}$ satisfies:

$$
\begin{gathered}
\text { For } v_{i_{0}}=\min _{i} v_{i}, \quad \Delta_{h} v_{i_{0}} \geqslant 0 \text { and } \\
\text { for } v_{i_{0}}=\max v_{i}, \quad \Delta_{h} v_{i_{0}} \leqslant 0 .
\end{gathered}
$$

This property holds for all schemes such that $b_{i i}=\sum_{k \neq i}\left|b_{k i}\right|$ and $b_{k i}<0$, since $\Delta_{h} v_{i}=\sum_{k} b_{k i} v_{k}=\sum_{k \neq i}\left|b_{k i}\right|\left(v_{i}-v_{k}\right)$. In particular, for the usual one-dimensional discrete Laplacian,

$$
\begin{gathered}
b_{i, i-1}=-2 /\left(h_{i}+h_{i+1}\right) h_{i}, \quad b_{i, i+1}=-2 /\left(h_{i}+h_{i+1}\right) h_{i+1} \quad \text { and } \\
b_{i, i}=-b_{i, i-1}-b_{i, i+1} .
\end{gathered}
$$

LEMMA 3.2. Let $\left\{u_{h}^{n}\right\}_{n \geqslant 1}$ be the solution of $\left(\mathrm{P}_{h}\right)$, where $\Delta_{h}$ satisfies property (L). Also let $b=(1-a k)^{-1}, \bar{u}^{m}=\max _{i} u_{i}^{m}$ and $\underline{u}^{m}=\min _{i} u_{i}^{m}$. Then

(a) For $\underline{u}^{n-1} \geqslant 0,0 \leqslant \underline{u}^{n} \leqslant \bar{u}^{n} \leqslant b \bar{u}^{n-1}$;

(b) For $\underline{u}^{n-1} \leqslant 0, b \underline{u}^{n-1} \leqslant \underline{u}^{n} \leqslant \bar{u}^{n} \leqslant 0$;

(c) For $\underline{u}^{n-1}<0$ and $\bar{u}^{n-1}>0, b \underline{u}^{n-1} \leqslant \underline{u}^{n} \leqslant \bar{u}^{n} \leqslant b \bar{u}^{n-1}$.

Proof. Let $u_{i_{0}}^{n}=\bar{u}^{n}$; then as $f$ is monotone nondecreasing, $f\left(u_{i_{0}}^{n}\right) \geqslant f\left(u_{i}^{n}\right)$, hence $\Delta_{h} f\left(u_{i_{0}}^{n}\right) \leqslant 0$, and it follows that

$$
(1-a k) u_{i_{0}}^{n}+k g\left(u_{i_{0}}^{n}\right)=u_{i_{0}}^{n-1}+k \Delta_{h} f\left(u_{i_{0}}^{n}\right) \leqslant \bar{u}^{n-1} .
$$

Similarly, letting $i_{0}$ be such that $u_{i_{0}}^{n}=\underline{u}^{n}$, we obtain $\underline{u}^{n-1} \leqslant(1-a k) \underline{u}^{n}+k g\left(\underline{u}^{n}\right)$. As $g$ is monotone with $g(0)=0$, we have $\operatorname{sign} g(x)=\operatorname{sign}(x)$; hence (a), (b), and (c) follow by considering the different cases.

4. Convergence Results. Our main convergence result is for smooth initial data $u_{0}$. The density of $D(A)$ and the fact that the operators $J_{\lambda}, J_{\lambda}^{h}$, and $S(t)$ are Lipschitz continuous immediately yield convergence for any $u_{0} \in L^{1}(\Omega)$.

TheOREM 4.1. Let $u_{0} \in C^{0, \alpha \beta}(\bar{\Omega}) \cap D(A), f\left(u_{0}\right) \in C^{2}(\bar{\Omega})$, and $\Delta t=t / m, t$ fixed. Then for $u_{h}^{m}$ computed with scheme $\left(\mathrm{P}_{h}\right)$,

$$
\left\|S(t) u_{0}-p_{h} u_{h}^{m}\right\|_{1}=O\left(\Delta t^{1 / 2}\right)+O\left(h^{\alpha \beta /(3+2 \alpha)}\right) .
$$

Remark. As will be apparent from the proof, we can eliminate the assumption $f\left(u_{0}\right) \in C^{2}(\bar{\Omega})$ and improve the constants in (4.1) by taking $\Delta t=h^{2 \alpha \beta /(3+2 \alpha)}$. Here, $\Delta_{h}$ is any diagonal dominant scheme consistent with the Laplacian in the sense that for $v \in C^{2, \alpha \beta}(\bar{\Omega})$ and $x$ in $\Omega,\left|\Delta_{h} v(x)-\Delta v(x)\right| \leqslant$ const $\cdot\|v\|_{C^{2, \alpha \beta}(\bar{\Omega})} h^{\alpha \beta}$.

Proof of Theorem 4.1. Assume $a=0$. We recall that if $B \in \mathscr{A}(a)$ and $R(I+\lambda B)$ $\supseteq \overline{D(B)}$ for any $0<\lambda<a^{-1}$, then for any $x \in D(B)$ (cf. (1.10) in [8]),

$$
\begin{aligned}
& \left\|S(t) x-J_{t / n}^{n} x\right\| \leqslant 2 t n^{-1 / 2} e^{4 a t}\|B x\|, \\
& \left\|J_{t / m}^{m} x-J_{t / n}^{n} x\right\| \leqslant 2 t e^{4 a t}|1 / m-1 / n|^{1 / 2}\|B x\| .
\end{aligned}
$$


From the above, and the fact that $S(t), J_{\lambda}$, and $p_{h}$ are contractions, we obtain

$$
\begin{aligned}
\| S(t) & u_{0}-p_{h} u_{h}^{m} \|_{1} \\
\leqslant & \left\|S(t) u_{0}-J_{t / m}^{m} u_{0}\right\|_{1}+\left\|J_{t / m}^{m} u_{0}-J_{t / n}^{n} u_{0}\right\|_{1} \\
& +\left\|J_{t / n}^{n} u_{0}-p_{h}\left(J_{t / n}^{h}\right)^{n} r_{h} u_{0}\right\|_{1}+\left\|\left(J_{t / n}^{h}\right)^{n} r_{h} u_{0}-\left(J_{t / m}^{h}\right)^{m} r_{h} u_{0}\right\|_{h} \\
\leqslant & 2 t\left((1 / m)^{1 / 2}+|1 / m-1 / n|^{1 / 2}\right)\left\|A u_{0}\right\|_{1}+2 t|1 / n-1 / m|^{1 / 2}\left\|A_{h} r_{h} u_{0}\right\|_{h} \\
& +\left\|J_{t / n}^{n} u_{0}-p_{h} r_{h} J_{t / n}^{n} u_{0}\right\|_{1}+\left\|r_{h} J_{t / n}^{n} u_{0}-\left(J_{t / n}^{h}\right)^{n} r_{h} u_{0}\right\|_{h} .
\end{aligned}
$$

For notational convenience we drop the $t / n$ subscript. Repeatedly using that $J^{h}$ is a contraction, for the last term in (4.2) we obtain

$$
\begin{aligned}
& \left\|r_{h} J^{n} u_{0}-\left(J^{h}\right)^{n} r_{h} u_{0}\right\|_{h} \\
& \leqslant\left\|r_{h} J\left(J^{n-1} u_{0}\right)-J^{h} r_{h}\left(J^{n-1} u_{0}\right)\right\|_{h}+\left\|r_{h} J^{n-1} u_{0}-\left(J^{h}\right)^{n-1} r_{h} u_{0}\right\|_{h} \\
& \quad \leqslant n \max _{0 \leqslant s \leqslant n-1}\left\|r_{h} J\left(J^{s} u_{0}\right)-J^{h} r_{h}\left(J^{s} u_{0}\right)\right\|_{h} .
\end{aligned}
$$

By definition, $v:=J\left(J^{s} u_{0}\right)$ and $v_{h}:=J^{h} r_{h}\left(J^{s} u_{0}\right)$ satisfy

$$
\begin{aligned}
& v_{h}+(t / n)\left[-\Delta_{h} f\left(v_{h}\right)+g\left(v_{h}\right)\right]=r_{h} J^{s} u_{0}, \\
& r_{h} v+(t / n)\left[-\Delta_{h} f\left(r_{h} v\right)+g\left(r_{h} v\right)\right] \\
& =r_{h} J^{s} u_{0}+(t / n)\left[r_{h} \Delta f(v)-\Delta_{h} f\left(r_{h} v\right)\right] .
\end{aligned}
$$

Here we used that since $r_{h}$ is a pointwise evaluation, $r_{h} f(v)=f\left(r_{h} v\right)$ and $r_{h} g(v)=$ $g\left(r_{h} v\right)$. Given that $A_{h}$ is accretive, from (4.4) we obtain

$$
\left\|r_{h} J\left(J^{s} u_{0}\right)-J^{h} r_{h}\left(J^{s} u_{0}\right)\right\|_{h} \leqslant(t / n)\left\|\Delta_{h} r_{h} f(v)-r_{h} \Delta f(v)\right\|_{h} .
$$

To complete our estimates, we need some regularity results. Given any $s, 0 \leqslant s \leqslant$ $n-1$, let $w_{s}$ be the solution of

$$
\gamma\left(w_{s}\right)-(t / n) \Delta w_{s}=J^{s} u_{0}, \quad w_{s} \in D(-\Delta)
$$

By Lemma 2.1, we have $\left\|\gamma\left(w_{s}\right)\right\|_{q} \leqslant\left\|J^{s} u_{0}\right\|_{q} \leqslant\left\|u_{0}\right\|_{q}$ for $1 \leqslant q \leqslant \infty$. First let $q>d$ and put $\varepsilon=1-d / q(>0)$. Since $w_{s}$ is a solution of the equation $-\Delta w_{s}=$ $(n / t)\left(J^{s} u_{0}-\gamma\left(w_{s}\right)\right)$ and $\Delta w_{s} \in L^{q}(\Omega)$, it follows that $w_{s} \in W^{2, q}(\Omega) \cap W_{0}^{1, q}(\Omega)$. But $W^{2, q}(\Omega)$ is embedded in $C^{1, \varepsilon}(\bar{\Omega})$, and so there is a positive number $K=$ $K(\Omega, d, q)$, such that

$$
\begin{aligned}
\left\|w_{s}\right\|_{C^{1, \ell}(\bar{\Omega})} & \leqslant K\left\|\Delta w_{s}\right\|_{q}=K(n / t)\left\|J^{s} u_{0}-\gamma\left(w_{s}\right)\right\|_{q} \\
& \leqslant K \cdot(n / t) \cdot 2\left\|u_{0}\right\|_{q}=\operatorname{const}(n / t) .
\end{aligned}
$$

Thus, in particular, $\left\|w_{s}\right\|_{C^{1}(\bar{\Omega})} \leqslant$ const $\cdot(n / t)$. Next, we show that $\left\|w_{s}\right\|_{C^{2, \alpha \beta}(\bar{\Omega})} \leqslant$ const $\cdot(n / t)^{1+\alpha}$. Since $w_{s} \in C^{1}(\bar{\Omega})$ for $s$ with $0 \leqslant s \leqslant n-1$, we have

$$
J^{s} u_{0} \equiv f^{-1}\left(w_{s-1}\right) \in C^{0, \alpha}(\bar{\Omega}) \text { for } 1 \leqslant s \leqslant n
$$

and

$$
\gamma\left(w_{s}\right) \equiv f^{-1}\left(w_{s}\right)+(t / n) g\left(f^{-1}\left(w_{s}\right)\right) \in C^{0, \alpha \beta}(\bar{\Omega}) \text { for } 1 \leqslant s \leqslant n .
$$

Hence,

$$
-\Delta w_{s}=(n / t)\left(J^{s} u_{0}-\gamma\left(w_{s}\right)\right) \in C^{0, \alpha \beta}(\bar{\Omega})
$$


Noting that $\Delta$ is considered in the bounded domain $\Omega$ under the Dirichlet condition, we apply the usual Hölder estimates to obtain

$$
\begin{aligned}
\left\|w_{s}\right\|_{C^{2, \alpha \beta}(\bar{\Omega})} & \leqslant \text { const } \cdot\left\|\Delta w_{s}\right\|_{C^{0, \alpha \beta}(\bar{\Omega})} \\
& \leqslant \text { const } \cdot(n / t)\left[\left\|J^{s} u_{0}\right\|_{C^{0, \alpha \beta}(\bar{\Omega})}+\left\|\gamma\left(w_{s}\right)\right\|_{C^{0 . \alpha \beta}(\bar{\Omega})}\right] .
\end{aligned}
$$

We then demonstrate that both $\left\|J^{s} v_{0}\right\|$ and $\left\|\gamma\left(w_{s}\right)\right\|$ are bounded by const $\cdot(n / t)^{\alpha}$. Since

$$
\begin{aligned}
\left|f^{-1}\left(w_{s-1}(x)\right)\right| & \leqslant \text { const } \cdot\left|w_{s-1}(x)\right|^{\alpha} \leqslant \text { const } \cdot\left\|w_{s-1}\right\|_{L^{\infty}(\Omega)}^{\alpha} \\
& \leqslant \text { const } \cdot\left\|w_{s-1}\right\|_{C^{1}(\bar{\Omega})}^{\alpha} \leqslant \text { const } \cdot(n / t)^{\alpha}
\end{aligned}
$$

and

$$
\begin{aligned}
& \left|f^{-1}\left(w_{s-1}(x)\right)-f^{-1}\left(w_{s-1}(y)\right)\right| \leqslant\left\|f^{-1}\right\|_{C^{0 . \alpha}(\bar{\Omega})} \cdot\left|w_{s-1}(x)-w_{s-1}(y)\right|^{\alpha} \\
& \quad \leqslant\left\|f^{-1}\right\|_{C^{0 . \alpha}(\bar{\Omega})}\left(\left\|w_{s-1}\right\|_{C^{1}(\bar{\Omega})} \cdot|x-y|\right)^{\alpha} \leqslant \mathrm{const} \cdot(n / t)^{\alpha} \cdot|x-y|^{\alpha}
\end{aligned}
$$

we have

$$
\left\|J^{s} v_{0}\right\|_{C^{0, \alpha \beta}(\bar{\Omega})}=\left\|f^{-1}\left(w_{s-1}\right)\right\|_{C^{0, \alpha \beta}(\bar{\Omega})} \leqslant \text { const } \cdot\left\|f^{-1}\left(w_{s-1}\right)\right\|_{C^{0, \alpha}(\bar{\Omega})} \leqslant \text { const } \cdot(n / t)^{\alpha} .
$$

Similarly, we obtain

$$
\begin{aligned}
\left\|\gamma\left(w_{s}\right)\right\|_{C^{0 . \alpha \beta}(\bar{\Omega})} & \leqslant\left\|f^{-1}\left(w_{s}\right)\right\|_{C^{0 . \alpha \beta}(\bar{\Omega})}+(t / n)\left\|g\left(f^{-1}\left(w_{s}\right)\right)\right\|_{C^{0 . \alpha \beta}(\bar{\Omega})} \\
& \leqslant \text { const } \cdot(n / t)^{\alpha}+\text { const } \cdot(t / n) \cdot(n / t)^{\alpha} .
\end{aligned}
$$

Noting that $t$ is a fixed positive number and $n$ is sufficiently large, we conclude that $n / t>1$ and $\left\|\gamma\left(w_{s}\right)\right\|_{C^{0 . \alpha \beta}(\bar{\Omega})} \leqslant$ const $\cdot(n / t)^{\alpha}$. Consequently, it follows that

$$
\left\|w_{s}\right\|_{C^{2, \alpha \beta}(\bar{\Omega})} \leqslant \text { const } \cdot(n / t)^{1+\alpha}
$$

holds for $n$ sufficiently large.

In (4.2) and (4.5) we have $J^{n} u_{0} \equiv f^{-1}\left(w_{n-1}\right)$ and $f(v) \equiv w_{s}$; consequently from (4.6), taking $(t / n)=h^{r}$, we obtain

$$
\begin{gathered}
\left\|\Delta_{h} r_{h} w_{s}-r_{h} \Delta w_{s}\right\|_{h} \leqslant \text { const } \cdot\left\|w_{s}\right\|_{C^{2, \alpha \beta}(\bar{\Omega})} h^{\alpha \beta} \leqslant \text { const } \cdot h^{\alpha \beta-r(1+\alpha)}, \\
\left\|J^{n} u_{0}-p_{h} r_{h} J^{n} u_{0}\right\|_{1} \leqslant \text { const } \cdot\left\|f^{-1}\left(w_{n-1}\right)\right\|_{C^{0 . \alpha}(\bar{\Omega})} h^{\alpha} \leqslant \text { const } \cdot h^{\alpha-r \alpha} .
\end{gathered}
$$

Substituting back in (4.5), (4.3), and (4.2), since

$$
h^{r / 2}=(t / n)^{1 / 2} \leqslant(t / m)^{1 / 2}+|(t / n)-(t / m)|^{1 / 2} \leqslant \Delta t^{1 / 2}+h^{r / 2},
$$

it is easy to see that the best choice of $r$ is $r=2 \alpha \beta /(3+2 \alpha)$, thus yielding (4.1). For the case $a>0$, only some $t$-dependent coefficients would have to be included in the inequalities (see [25]).

5. Berger et al. Algorithms. Let $R(t)$ denote the semigroup generated by $-\Delta$ in $L^{1}(\Omega)$. Berger et al. [4] approximate (P) for $g(u) \equiv 0$ and $a=0$ by

$$
\left(u^{n+1}-u^{n}\right) / k+\frac{1}{\sigma}(I-R(\sigma)) f\left(u^{n}\right)=0, \quad u^{0}=u_{0},
$$

where $\sigma$ is a positive function of $k$ satisfying $\lim _{k \rightarrow 0} \sigma=0$. From it we obtain the "analytical" form of the algorithm,

$$
u^{n+1}=u^{n}+\frac{k}{\sigma}\left[R(\sigma) f\left(u^{n}\right)-f\left(u^{n}\right)\right], \quad u^{0}=u_{0},
$$


which involves no spatial discretization. For $k=t / n, t$ fixed, convergence of $u^{n}$ to $S(t) u_{0}$ was established in [4], using approximation results in [6], under the assumptions that $u_{0} \in L^{\infty}(\Omega), f$ is locally Lipschitz continuous with constant $\mu$ on $\left[-\left\|u_{0}\right\|_{\infty},\left\|u_{0}\right\|_{\infty}\right]$, and $\mu k \leqslant \sigma$.

The numerical schemes are obtained by discretizing the linear heat problem $R(\sigma) f\left(u^{n}\right)$, and are given by

$$
u_{h}^{n+1}=T(k) u_{h}^{n}, \quad u_{h}^{0}=r_{h} v_{0},
$$

where $T(k)$ is defined by

$$
T(k) v_{h}:=v_{h}+\frac{k}{\sigma}\left[K_{\sigma}^{h} f\left(v_{h}\right)-f\left(v_{h}\right)\right],
$$

and $K_{\sigma}^{h}$ is one of the usual implicit finite-difference operators for the heat equation, i.e.,

$$
K_{\sigma}^{h}=\left(I-\theta \sigma \Delta_{h}\right)^{-1}\left(I+(1-\theta) \sigma \Delta_{h}\right)
$$

for some $0<\theta \leqslant 1$.

Our convergence results allow us to prove convergence of the fully discretized (5.1)-(5.2) schemes under the above or other similar conditions. It is enough to consider the fully implicit scheme, and the proof consists of (a) establishing that $(I-T(k)) / k$ is $m$-accretive in $l_{h}^{1}$, (b) showing that $T(k)$ is Lipschitz continuous, (c) using arguments like those in Section 4, and (d) applying Lemma $4^{\prime}$ in [22] together with (4.1). See [25] for details.

6. Numerical Experiments. We consider the one-dimensional problem with $f(u) \equiv$ $u^{2}$ and $g \equiv 0$. Barenblatt [2] and Pattle [23] established the exact weak solution when $f(u) \equiv u^{m}$ and the initial data is the Dirac delta; in this case it is given by

$$
u(x, t)= \begin{cases}\left(1-\left[x /(9 t)^{1 / 3}\right]^{2}\right) / \frac{4}{3}(9 t)^{1 / 3} & \text { for }|x| \leqslant(9 t)^{1 / 3} \\ 0 & \text { elsewhere }\end{cases}
$$

which shows that the interfaces are at $x= \pm(9 t)^{1 / 3}$.

The initial data for the numerical computations were taken to be the values at gridpoints of $u_{0}(x)$, the above solution at $t=1$. The grids were uniform with $\Delta x=L / N$, where $L=(9)^{1 / 3}$, and $N+1$ is the number of points in $[0, L]$, since we exploited the problem's symmetry. We took $k=\sqrt{\Delta x}$, to be able to neglect the effect of the space discretization, and consider the error $E$ in $l_{h}^{1}$ as $E=c k^{\rho}$.

From the algorithms of [4] we chose the totally implicit one with $k / \sigma=$ $\max \left\{f^{\prime}(z): z \in\left[-\left\|u_{0}\right\|_{\infty},\left\|u_{0}\right\|_{\infty}\right]\right\}=(3 / 8)^{1 / 3}$ and computed over the fixed interval $[0,2 L]$; we shall refer to it as BBR.

The nonlinear equations of $\left(\mathrm{P}_{h}\right)$ were solved with Newton's method, taking the solution in the previous time step as initial guess; we shall refer to this algorithm as NLN. Since the support of the numerical solution advances one gridpoint per Newton iteration, our initial set of equations corresponded to the gridpoints in $[0, L]$ and we added one equation per iteration. Also, we only iterated over those equations for which the relative error in the previous iteration $m$ was greater than $\left[.01(\Delta x)^{2} / \max u_{i}^{(m)}\right]$. For the following time step, only the new gridpoints at which the solution was greater than $10^{-25}$ were added to the support, thus determining the location of the numerical interface. With the above implementation, NLN was 
slightly faster than BBR, offsetting its advantage of only solving a linear system. Table 1 contains the errors and numerical rates of convergence to the exact solution at $t=4.2250$.

TABLE 1

\begin{tabular}{|c||l|l|l|l|l|l|}
\hline \multirow{2}{*}{$N$} & \multirow{2}{*}{$\Delta x$} & $k$ & \multicolumn{2}{c|}{ BBR } & \multicolumn{2}{c|}{ NLN } \\
\hline 20 & .1040 & .3225 & .1574 & & .00880 & \\
80 & .0260 & .1612 & .1100 & .5160 & & 1.0697 \\
320 & .0065 & .0806 & .07018 & .6492 & .00419 & \\
1280 & .00162 & .0403 & .04150 & & .00103 & \\
\hline
\end{tabular}

For this example, NLN was more accurate than BBR, particularly around the interface which at $t=4.225$ is at 3.3627. Table 2 contains, for both algorithms, the location of the smallest value greater than $10^{-20}$, and for NLN the first point at which the solution is less than 0.00005 , which computationally might be considered as zero, since the values at the flat part of the solution are close to 0.2 .

TABLE 2

\begin{tabular}{|c|c|c|c|c|cc|}
\hline & \multicolumn{2}{|c|}{ BBR } & \multicolumn{4}{c|}{ NLN } \\
$N$ & $x$ & VALUE & $x$ & VALUE & $x$ & VALUE \\
\hline 20 & 4.160 & $1.30(-3)$ & 3.7441 & $3.07(-11)$ & 3.6401 & $1.25(-6)$ \\
80 & 4.160 & $4.52(-4)$ & 3.5101 & $4.80(-11)$ & 3.4581 & $4.60(-5)$ \\
320 & 4.160 & $1.18(-4)$ & 3.4321 & $1.40(-14)$ & 3.4126 & $4.39(-5)$ \\
1280 & 4.160 & $2.39(-5)$ & 3.3899 & $6.07(-5)$ & 3.3899 & $6.07(-5)$ \\
& & & & & & \\
\hline
\end{tabular}

Acknowledgment. The author wishes to thank Professor R. C. MacCamy for his suggestions and lengthy hours of discussion, and he is also grateful to the referees for their suggestions which allowed him to improve the results and presentation of this paper.

Department of Mathematics

Carnegie-Mellon University

Pittsburgh, Pennsylvania 15213

1. V. Barbu, Nonlinear Semigroups and Differential Equations in Banach Spaces, Noordhoff, Leyden, 1976.

2. G. I. BarenblatT, "On certain non-stationary motions of liquids and gases in porous media," Prikl. Mat. Mekh., v. 16, 1952, pp. 67-78.

3. Ph. Benilan, Équations d'Évolution dans un Espace de Banach quelconque et Applications, Thesis, Univ. Paris XI, Orsay, 1972.

4. A. E. Berger, H. Brezis \& J. C. W. Rogers, "A numerical method for solving the problem $u_{t}-\Delta f(u)=0$," RAIRO Numer. Anal., v. 13, 1979, pp. 297-312.

5. H. Brezis, M. Crandall \& A. Pazy, "Perturbations of nonlinear maximal monotone sets in Banach spaces," Comm. Pure Appl. Math., v. 23, 1970, pp. 123-144. 
6. H. Brezis \& A. PAZY, "Convergence and approximation of semigroups of nonlinear operators in Banach spaces," J. Funct. Anal., v. 9, 1972, pp. 63-74.

7. H. Brezis \& W. A. Strauss, "Semi-linear second order elliptic equations in $L^{1}$," J. Math. Soc. Japan, v. 25, 1973, pp. 565-590.

8. M. Crandall \& T. LiggetT, "Generation of semi-groups of nonlinear transformations on general Banach spaces," Amer. J. Math., v. 93, 1971, pp. 265-298.

9. M. Crandall \& A. Pazy, "Nonlinear evolution equations in Banach spaces," Israel J. Math., v. 11,1972 , pp. 57-94.

10. M. CRANDall, "Semigroups of nonlinear transformations in Banach spaces," in Contributions to Nonlinear Functional Analysis (E. H. Zarantonello, ed.), Academic Press, New York, 1971, pp. 157-179.

11. J. Descloux, "On the equation of Boussinesq," in Topics in Numerical Analysis (J. J. H. Miller, ed.), Vol. 3, Academic Press, London, 1977, pp. 81-102.

12. J. I. Diaz Diaz, "Solutions with compact support for some degenerate parabolic problems," Nonlinear Anal., v. 3, 1979, pp. 831-847.

13. E. DiBenedetro \& D. C. HofF, "An interface tracking algorithm for the porous medium equation," Trans. Amer. Math. Soc., v. 284, 1984, pp. 463-500.

14. L. C. Evans, Nonlinear Evolution Equations, MRC, TSR No. 1568, 1975.

15. L. C. Evans, “Differentiability of a nonlinear semigroup in $L^{1}$," J. Math. Anal. Appl., v. 60, 1977, pp. $703-715$.

16. D. Gilbarg \& N. S. Trudinger, Elliptic Partial Differential Equations of Second Order, SpringerVerlag, New York, 1977.

17. M. E. Gurtin \& R. C. MacCamy, "On the diffusion of biological populations," Math. Biosci., v. 33, 1977, pp. 35-49.

18. M. E. Gurtin, R. C. MacCamy \& E. A. Socolovsky, "A coordinate transformation for the porous media equation that renders the free-boundary stationary," Quart. Appl. Math., v. 42, 1984, pp. 345-357.

19. R. C. MacCamy \& E. A. Socolovsky, "A numerical procedure for the porous media equation," Comput. Math. Appl., v. 11, 1985, pp. 315-319.

20. R. H. MARTin, JR., “A global existence theorem for autonomous differential equations in a Banach space," Proc. Amer. Math. Soc., v. 26, 1970, pp. 307-314.

21. M. Mimura, R. NAKAKI \& K. TomoedA, "A numerical approach to interface curves for some nonlinear diffusion equations," Japan J. Appl. Math., v. 1, 1984, pp. 93-139.

22. I. MiYadera \& S. OHARU, "Approximation of semigroups of nonlinear operators," Tôhoku Math. J., v. 22, 1970, pp. 24-47.

23. R. C. Pattle, "Diffusion from an instantaneous point source with a concentration-dependent coefficient," Quart. J. Mech. Appl. Math., v. 12, 1959, pp. 407-409.

24. M. E. Rose, “Numerical methods for flows through porous media. I,” Math. Comp., v. 40, 1983, pp. 435-467.

25. E. A. Socolovsky, On Numerical Methods for Degenerate Parabolic Problems, Thesis, CarnegieMellon University, August, 1984. 